\title{
3D model atmospheres and the solar photospheric oxygen abundance
}

\author{
E. Caffau ${ }^{1}$ and H.-G. Ludwig ${ }^{1,2}$ \\ ${ }^{1}$ GEPI, Observatoire de Paris-Meudon; 92195 Meudon, France \\ email: Elisabetta Caffau@obspm.fr \\ ${ }^{2}$ CIFIST Marie Curie Excellence Team \\ Observatoire de Paris-Meudon, 92195 Meudon, France \\ email: Hans.Ludwig@obspm.fr
}

\begin{abstract}
In recent years the photospheric solar oxygen abundance experienced a significant downward revision. However, a low photospheric abundance is incompatible with the value in the solar interior inferred from helioseismology. For contributing to the dispute whether the solar oxygen abundance is "high" or "low", we re-derived its photospheric abundance independently of previous analyses. We applied 3D (CO5BOLD) as well as 1D model atmospheres. We considered standard disc-centre and disc-integrated spectral atlases, as well as newly acquired solar intensity spectra at different heliocentric angles. We determined the oxygen abundances from equivalent width and/or line profile fitting of a number of atomic lines. As preliminary result, we find an oxygen abundance in the range 8.73-8.79, encompassing the value obtained by Holweger (2001), and somewhat higher than the value obtained by Asplund et al. (2005).
\end{abstract}

Keywords. Sun: abundances - Sun: photosphere - Line: formation - hydrodynamics

\section{Introduction}

After hydrogen and helium oxygen is the most abundant element in the Universe, and its abundance has been extensively studied in the Galaxy and beyond. In such studies the solar oxygen abundance serves as natural reference. However, the spectroscopic determination of the solar oxygen abundance is not an easy task, and unfortunately its meteoritic abundance cannot be used for guidance since oxygen - as rather volatile element - was incompletely condensed during the cooling of the proto-solar nebula. Much effort has been devoted to spectroscopic determination of the photospheric oxygen abundance without having led to a convergence on a definite value. This has repercussions on solar and stellar physics. For instance, oxygen is a major contributor to the opacity in the convective envelope of the Sun. Solar oxygen abundance has a direct impact on the internal structure and evolution of the Sun and solar-like stars.

Over the last twenty years the photospheric oxygen abundance has experienced a downward trend, brought only to a halt by a recent analysis of Ayres and collaborators of both $\mathrm{CO}$ molecular and [OI] forbidden lines. Some milestone results that we would like to point out are: $8.93 \pm 0.035$ in Anders \& Grevesse (1989), $8.83 \pm 0.06$ in Grevesse \& Sauval (1998), 8.736 \pm 0.078 in Holweger (2001), and 8.66 \pm 0.06 in Asplund et al. (2004). Despite oxygen is such an abundant element, very few atomic lines are available to determine the photospheric oxygen abundance.

\section{Data analysis}

We considered various high-resolution, high signal-to-noise $(\mathrm{S} / \mathrm{N})$ solar atlases for the oxygen abundance determination. Notably, the difference between them is higher than 


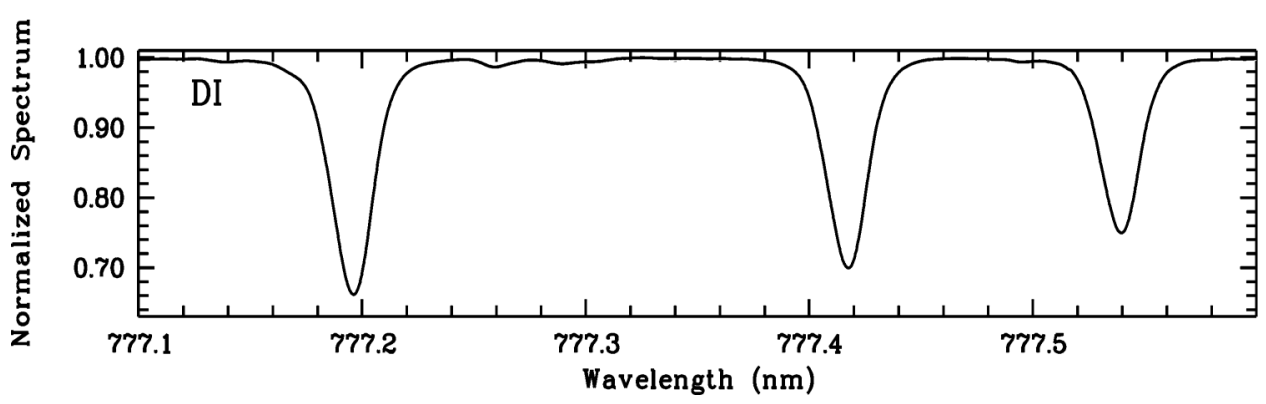

Figure 1. Comparison of two observed disc-centre spectra.

Table 1. Oxygen lines considered in this analysis

\begin{tabular}{llc}
\hline Wavelength & Characteristics & Number of spectra \\
\hline$(\mathrm{nm})$ & & \\
630.0 & blended with NiI line & 4 \\
636.3 & on CaI autoionisation line, blended with CN & 4 \\
777.1 & affected by strong NLTE correction & 4 \\
777.4 & affected by strong NLTE correction & 4 \\
777.5 & affected by strong NLTE correction & 4 \\
615.8 & weak, next to a strong line & 4 \\
844.6 & blended with a stronger FeI line & 1 \\
926.6 & blended with a telluric absorption & 1 \\
1130.2 & blended with a telluric absorption & 1 \\
1316.5 & blended with a telluric absorption & \\
\hline
\end{tabular}

expected according to the nominal signal-to-noise level, usually on the order of a few thousand. An example is shown in figure 1 where the grating disc-centre spectrum of Delbouille et al. (1973) (solid black line) is compared to the Fourier-transform spectrum of Neckel \& Labs(1984) (solid green/grey line) for the triplet lines. The differences in the EW of the three lines between the two spectra are 4, 7, and $3 \%$ in order of increasinf wavelength, which translates in abundance differences of $0.05,0.07$, and 0.03 dex, respectively.

The lines we considered are listed in Table 1.

As model of the solar atmosphere we used a $3 \mathrm{D}$ radiation-hydrodynamical model atmosphere (computed with the $\mathrm{CO}^{5} \mathrm{BOLD}$ code) which provides a statistical realisation of the evolution of the plasma flow in a volume located at the solar surface comprising optically thick and thin regions. $\mathrm{CO}^{5}$ BOLD solves simultaneously the hydrodynamical equation coupled to radiative transfer in an external gravity field. The particular solar model we used in the present analysis covers a time interval of $4300 \mathrm{~s}$ represented by a selection of 19 snapshots.

For spectral-synthesis purposes we used the code Linfor3D. Linfor3D is restricted to local thermodynamic equilibrium (LTE) conditions. Since departures from LTE are important for the determination of the solar oxygen abundance, in addition we performed NLTE calculations with the Kiel code (Steenbock \& Holweger (1984)) using the oxygen model atom from Paunzen et al. (1999). This Kiel code, however, is restricted to 1D structures. For this reason we used as input model the time and horizontal average of the 3D model over surfaces of equal optical depth. It has been shown (Asplund et al. (2004)) that the NLTE problem in the Sun is essentially a 1D problem, i.e., horizontal inhomogeneities play a minor role so that we consider the 1D approximation sufficient. 


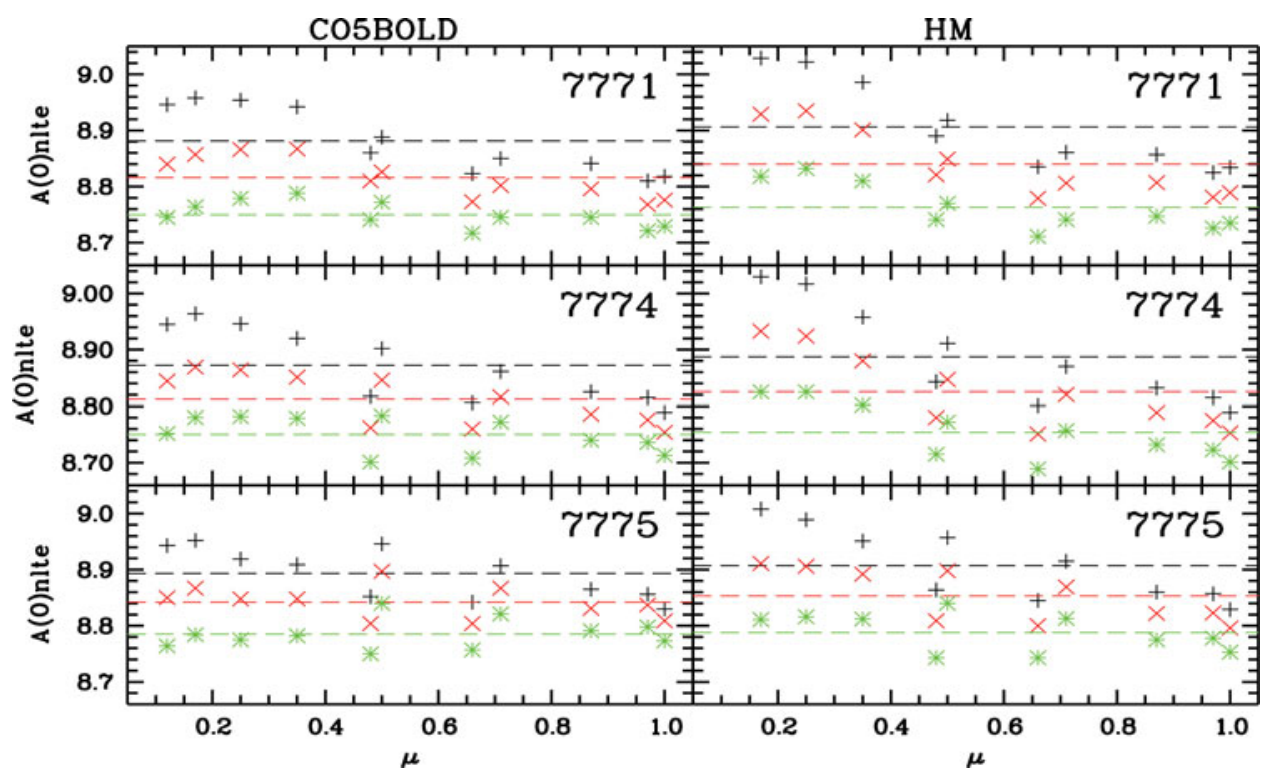

Figure 2. Oxygen abundance from the $777 \mathrm{~nm}$ triplet of centre-limb data.

In the Kiel code the excitation and ionisation of oxygen atoms by collisions with neutral hydrogen atoms is taken into account within the approximation provided by the Drawin (Drawin 1969) formula. A scaling factor, $\mathrm{S}_{\mathrm{H}}$, permits to take into account these collision, $\left(\mathrm{S}_{\mathrm{H}}=1\right)$, to switch them off, $\left(\mathrm{S}_{\mathrm{H}}=0\right)$, or to consider a fraction of them. No atomic physics calculations or laboratory measurements are available to constrain $\mathrm{S}_{\mathrm{H}}$. We tried to estimate its value by considering the centre-to-limb variation of the infrared triplet lines, similar to the work of Allende Prieto et al. (2004). Figure 2 depicts the oxygen abundance as a function of the cosine of the heliocentric angle $\mu$ for the three triplet components. We think we can exclude $\mathrm{S}_{\mathrm{H}}=1$ since it is the one that gives the much higher scatter of the oxygen abundance for various $\mu$ than the lower test values.

Considering 31 line/observed spectrum combinations, using the CO5BOLD 3D model, with the choice of $\mathrm{S}_{\mathrm{H}}=1 / 3$ in the NLTE computation we obtain $\mathrm{A}(\mathrm{O})=8.76 \pm 0.07$. The error includes random error related to scatter between the results obtained from different spectra, uncertainties in the continuum placement, the oscillator strength uncertainty, blends, and the choice of $\mathrm{S}_{\mathrm{H}}$ in the NLTE computation. This oxygen abundance translates into $\mathrm{Z}=0.014$ if we consider our value for oxygen abundance and the Asplund et al. (2005) abundances for the other elements, $Z=0.016$ when the other abundances are from Grevesse \& Sauval (1998). By comparison, the Asplund et al. (2005) abundances, including oxygen, imply $\mathrm{Z}=0.012$.

To quantify in how far 3D effects are important we performed a comparison with abundances obtained from a 1D model. We choose a 1D model computed with the code LHD which is a standard 1D model atmosphere code, sharing the same micro-physics with $\mathrm{CO}^{5} \mathrm{BOLD}$. The convective energy transport is treated with mixing-length theory. For the mixing-length parameter, $\alpha$, we used a value of 1.0. In the $1 \mathrm{D}$ spectral synthesis we adopted a micro-turbulence of $1 \mathrm{~km} / \mathrm{s}$. The choice of the mixing-length parameter is not important except for the $615.8 \mathrm{~nm}$ line, since all other oxygen lines are formed in layers whose temperature structure is virtually unaffected by differences in the assumed efficiency of convective energy transport. 


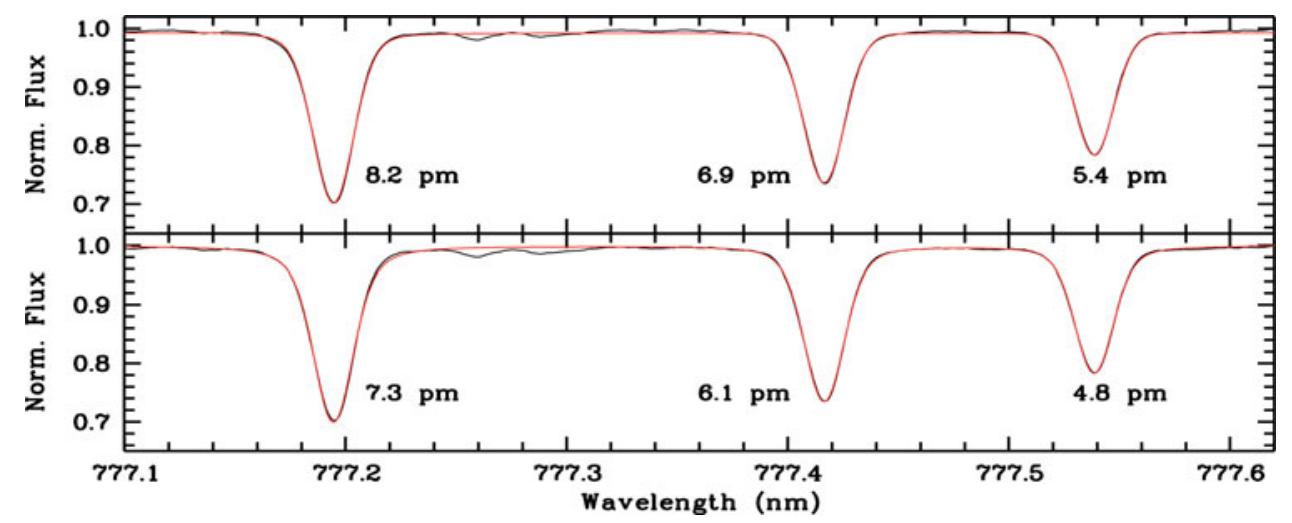

Figure 3. Differences in EW for the $777 \mathrm{~nm}$ triplet.

We define $3 \mathrm{D}$ correction the difference $\mathrm{A}(\mathrm{O})_{3 \mathrm{D}}-\mathrm{A}(\mathrm{O})_{\mathrm{LHD}}$, as in Caffau \& Ludwig (2007). The average $3 \mathrm{D}$ correction we found is +0.05 dex. For all lines except the $615.8 \mathrm{~nm}$ line, the $3 \mathrm{D}$ correction is positive. We conclude that $3 \mathrm{D}$ effects are not responsible for lowering the oxygen abundance. This conclusion obviously depends on our choice of the 1D model atmosphere we used as reference. We belief, however, that our particular choice of a differentially comparable model in terms of the intrinsic micro-physics provides a well-defined reference. Our higher oxygen abundance in comparison to Asplund et al. (2004) is in part related to the EWs measurements. The triplet lines are important in the $\mathrm{A}(\mathrm{O})$ determination, and their EWs are lower in Asplund et al. (2004) with respect to what we measure. In figure 3 we show the subjective component that is present in the placement of the continuum for the $777 \mathrm{~nm}$ triplet lines. The upper panel shows the Voigt profile to compute the EW as we did in this work, in the lower panel we tried to reproduce the EWs published in Asplund et al. (2004). We think that in this latter case part of the wings are lost.

We compared the oxygen abundance of the Sun with these of stars with and without detected planets. Ecuvillon et al. (2006) compared the oxygen abundance in a sample of 96 stars with planets to a volume limited sample of 59 stars without planets. The solar oxygen is very close to the mean oxygen abundance of the sample with planets $(0.01 \mathrm{dex}$ lower). The Asplund et al. (2004) value is very close to the mean oxygen abundance of the sample without planets. But both determinations of the solar oxygen abundance are compatible with the $\mathrm{A}(\mathrm{O})$ for stars hosting planets.

\section{References}

Allende Prieto, C., Asplund, M., \& Fabinani Bendicho, P. 2004, A $\mathscr{J} A$ 423, 1109

Anders, E. \& Grevesse, N. 1989, Geochim. Cosmochim. Acta 53, 197

Asplund, M. et al. 2004, A\&A 417, 751

Asplund, M., Grevesse, N., \& Sauval, A. J. 2005, ASP Conf. Ser. 336, 25

Caffau, E. \& Ludwig, H.-G. 2007, A\&A 467, L11

Delbouille, L., Roland, G., \& Neven, L. 1973, Liege: Universite de Liege, IA 1973

Drawin, H. W., 1969, Z. Physik 225, 483

Ecuvillon, A., et al. 2006, A\& A 445, 633

Grevesse, N. \& Sauval, A. J. 1998, Space Science Reviews 85, 161

Holweger, H. 2001, AIP Conf. Proc., 598, 23

Neckel, H. \& Labs, D. 1984, Sol. Phys. 90, 205

Paunzen, E., et al. 1999, A\&A 345, 597

Steenbock, W. \& Holweger, H. 1984, A\&SA 130, 319 


\section{Discussion}

Asplund: Two comments: (1) In Asplund et al. (2004) we don't use equivalent widths but line profile fitting in 3D non-LTE. (2) One has to be careful when comparing the Sun with the distribution of stars "with" and "without" planets. We don't know yet whether the "without planets" sample really don't have planets, only that they don't have "hot Jupiters" which the Sun of course does not have.

CAffau: (1) Thank you for clarification. (2) Yes. I do not think that the Solar oxygen abundance respect to the histogram of stars with or without "hot Jupiters" can anyway solve the problem. 